INPLASY

PROTOCOL

To cite: Li et al. Comparative efficacy of various pharmacologic treatments of alcohol withdrawal syndrome: A systematic review and network meta-analysis. Inplasy protocol 2021120010. doi: 10.37766/inplasy2021.12.0010

Received: 02 December 2021

Published: 02 December 2021

Corresponding author:

Gui-Ping Xu

xgpsyl@126.com

Author Affiliation:

Department of anesthesia, People's Hospital of Xinjiang Uygur Autonomous Region.

Support: NNSF of China.

Review Stage at time of this submission: Completed but not published.

Conflicts of interest: None declared.

\section{Comparative efficacy of various pharmacologic treatments of alcohol withdrawal syndrome: A systematic review and network meta-analysis}

\author{
Li, Q1; Ma, X²; Simayi, A3; Wang, X4; Xu, G5.
}

Review question / Objective: Lorazepam and other benzodiazepines (BZDs) are considered the first choice for treatment of Alcohol withdrawal syndrome (AWS). But they have significant addiction potential and can cause fatal respiratory depression if used in large doses. The aim of our study is to conduct a network meta-analysis to provide some data support for the clinical treatment of AWS. The patients were persons with alcohol withdrawal. The intervention being studied must be a comparison of the efficacy of the two pharmacologic treatments. The study should not be included if two pharmacologic treatments belonging to the same category were compared. All studies must include one of the following outcomes: Clinical Institute Withdrawal Assessment, revised (CIWA-Ar) score, length of hospital stay, length of intensive care unit (ICU) stay, and the incidence of delirium or seizures.

Condition being studied: Side effects and safety of eleven types of agents currently used to treat alcohol withdrawal syndrome.

INPLASY registration number: This protocol was registered with the International Platform of Registered Systematic Review and Meta-Analysis Protocols (INPLASY) on 02 December 2021 and was last updated on 02 December 2021 (registration number INPLASY2021120010).

\section{INTRODUCTION}

Review question / Objective: Lorazepam and other benzodiazepines (BZDs) are considered the first choice for treatment of Alcohol withdrawal syndrome (AWS). But they have significant addiction potential and can cause fatal respiratory depression if used in large doses. The aim of our study is to conduct a network meta-analysis to provide some data support for the clinical treatment of AWS. The patients were 
persons with alcohol withdrawal. The intervention being studied must be a comparison of the efficacy of the two pharmacologic treatments. The study should not be included if two pharmacologic treatments belonging to the same category were compared. All studies must include one of the following outcomes: Clinical Institute Withdrawal Assessment, revised (CIWA-Ar) score, length of hospital stay, length of intensive care unit (ICU) stay, and the incidence of delirium or seizures.

Rationale: Alcohol is one of the oldest and most widely consumed substances in the world. About 2 billion people consume alcoholic beverages worldwide. However, alcohol consumption is a serious global health problem with high morbidity and mortality. Alcohol withdrawal syndrome (AWS) is a complex and dynamic disease, mainly caused by an imbalance between the inhibitory neurotransmitter $\gamma$ aminobutyric acid (GABA) and the excitatory amino acid glutamate. Due to the inhibitory effects of alcohol, chronic alcohol use leads to a down-regulation of GABA receptors and a compensatory upregulation of $\mathrm{N}$-methyl-D-aspartate (NMDA) receptors, the binding sites of glutamate. In AWS, the inhibitory effect of alcohol is completely eliminated from the system, while excitatory glutamate floods the central nervous system (CNS). Currently, the main goal of treatment of AWS is to prevent the development of tremor and delirium by correcting this imbalance. The way of treatment of AWS includes pharmacologic intervention and assistance. Assistance includes caregiver support, elimination of stress stimuli (such as bright lights, noise), maintaining contact with reality and counseling. Pharmacological approaches, by contrast, include providing patients with general support to correct any biochemical imbalances (usually electrolyte imbalance, dehydration, hypoglycemia, and vitamin deficiency, especially group B and folic acid), as well as specific therapies for any disease. Lorazepam and other benzodiazepines (BZDs) are considered the first choice for treatment of AWS. BZDs are effective because they have an effect on the GABA-A receptor, which is lost in large quantities if alcohol is suddenly withdrawn. The effects of BZDs come not only from their metabolites, but also from themselves, thereafter hepatic glucuronidation inactivates BZD metabolites, which in turn are cleared by the kidney. While BZDs are effective at preventing life-threatening symptoms of AWS, they also have problems. Lorazepam has significant addiction potential and can cause fatal respiratory depression if used in large doses. In this case, clinicians are trying to find alternatives or supplements to BZDs. The main objective of pharmacological management of AWS is to minimize the severity of symptoms and prevent major complications. The ideal pharmacologic treatments should be cross-tolerated with alcohol. It should have rapid onset, wide safety, metabolism unrelated to liver function, low potential for alcohol use disorder, sedative, anxiolytic, and anticonvulsant effects without causing respiratory depression. Therefore, multiple combinations therapies or monotherapies suitable for AWS have been tried clinically, including alpha-adrenergic agonists, barbiturates, beta-blockers, butyrophenones, carbamazepine, dexmedetomidine, gabapentin, propofol and valproic acid. However, there is still much controversy about the efficacy and side effects of these pharmacologic treatments.

Condition being studied: Side effects and safety of eleven types of agents currently used to treat alcohol withdrawal syndrome.

\section{METHODS}

Search strategy: We searched articles published before January 31, 2020 in 4 electronic databases: PubMed, EMBASE, Web of Science and Cochrane Library. The articles were selected by manual screening. The following terms were used in the search: (alcohol withdrawal [Title]) OR (alcohol withdrawal delirium[MeSH Terms]) OR (alcohol withdrawal induced delirium tremens[MeSH Terms])) AND (Anticonvulsant OR Anticonvulsant OR anti- 
epileptic OR antiepileptic OR Baclofen OR Benzodiazepine OR Benzodiazepines OR barbiturates OR Bromocriptine OR Carbamazepine OR caroverine OR chlordiazepoxide OR Chlormethiazole OR clomethiazole OR clonazepam OR Clonidine OR Dexmedetomidine OR diazepam OR enteral ethanol OR flunitrazepam OR GABA agonists OR gabapentin OR Gamma-hydroxybutyrate OR haloperidol OR histamine antagonists OR hydroxyzine OR intravenous ethanol OR ketamine OR Levetiracetam OR lorazepam OR lofexidine OR mesoridazine OR meprobamate OR nitrous oxide OR nonGABA adjunctive agents OR Oxazepam OR oxcarbazepine OR phenobarbital OR propofol OR Sodium oxybate OR tetrabamate OR tiapride OR tranquilizing agent OR trazodone OR valproate OR valproic acid).

Participant or population: Patients with alcohol withdrawal syndrome.

Intervention: 1) BZDs: chlordiazepoxide, clonazepam, diazepam, lorazepam, oxazepam; 2) anticonvulsants: clomethiazole, carbamazepine, depakote, divalproex, gabapentin, levetiracetam, pregabalin, and zonisamide; 3) barbiturates: phenobarbital, and barbiturate; 4) antipsychotics: cyamemazine, and tiapride; 5) a2-ARagonists: dexmedetomidine, andclonidine.

Comparator: 1) BZDs: chlordiazepoxide, clonazepam, diazepam, lorazepam, oxazepam ; 2) anticonvulsants: clomethiazole, carbamazepine, depakote, divalproex, gabapentin, levetiracetam, pregabalin, and zonisamide; 3) barbiturates: phenobarbital, and barbiturate; 4) antipsychotics: cyamemazine, and tiapride; 5) a2-ARagonists: dexmedetomidine, andclonidine. 6) Placebo.

Study designs to be included: Randomized controlled trials (RCTs) and cohort studies.

Eligibility criteria: The patients were persons with alcohol withdrawal. The intervention being studied must be a comparison of the efficacy of the two pharmacologic treatments. The study should not be included if two pharmacologic treatments belonging to the same category were compared. All studies must include one of the following outcomes: Clinical Institute Withdrawal Assessment, revised (CIWA-Ar) score, length of hospital stay, length of intensive care unit (ICU) stay, and the incidence of delirium or seizures.

Information sources: We searched articles published before January 31, 2020 in 4 electronic databases: PubMed, EMBASE, Web of Science and Cochrane Library.

Main outcome(s): In order to conduct a pairwise comparison of the therapeutic effects between any two of 11 pharmacologic treatments or pharmacologic treatment combinations, this study defined the following three outcomes: 1) mean difference (MD) in mean change in CIWA-Ar score after treatment and before treatment; 2) mean difference in length of hospital stay and mean difference in length of ICU stay; and 3) Odds ratio (OR) of incidence of delirium or seizures.

Data management: Two investigators used a predefined data extraction sheet to independently extract data from each included study, such as authors, publication year, study design, sample size, grouping and number of patients in the group, baseline and endpoint data, including counts and effect estimates (mean $\pm \mathrm{sd}$ ), country, follow-up months, title, and conclusion. The third investigator independently reviewed the data to ensure accuracy. If a drug was administered at different dosages in different arms of a trial, these arms were combined. If no data in digital format were available, we used the free software Plot Digitizer to estimate data from the graphs.

Quality assessment / Risk of bias analysis: Each study was graded for potential bias into low, high and unclear according to the Cochrane Handbook of Systematic Reviews of Interventions, 5.1.0 (updated March 2011). Inconsistency was tested 
both globally using a chi-squared test and locally using a side-splitting test of inconsistency and loop-specific inconsistency plots. Finally, a comparisonadjusted funnel plot was used to assess small study effects within a network of interventions.

Strategy of data synthesis: We used the network meta-analysis approach to evaluate the comparative effect by combining direct and indirect evidence of 11 pharmacologic treatments. To visualize network geometry and node connectivity, we summarized the geometry of the evidence network using network plots. We used the frequentist framework to perform a random effect network meta-analysis. To obtain treatment hierarchies, we used a parametric bootstrap procedure with $\mathbf{5 0 0 0}$ resamples to compute ranking probabilities. Mean rankings as well as surface under the cumulative ranking curve (SUCRA) values were computed for each treatment.

Subgroup analysis: In order to avoid the heterogeneity of the merger of different study designs, we did not merge RCTs and cohort studies. Each outcome was divided into two subgroups for combined analysis, one was the RCTs subgroup and the other was the retrospective research subgroup.

\section{Sensitivity analysis: None}

Language: No language limit.

Country(ies) involved: China.

Keywords: Alcohol withdrawal syndrome; Benzodiazepine; Anticonvulsant; Dexmedetomidine; Barbiturates; Network meta-analysis.

Contributions of each author:

Author 1 - Qu Li - Conceptualization, Data curation, Formal analysis, Investigation, Methodology.

Email: quli1212@yeah.net

Author 2 - Xue-Ping Ma - Data curation, Investigation, Resources, Validation, Visualization, Writing-review \& editing.

Email: 1500431388@qq.com
Author 3 - Alimujiang Simayi - Data curation, Investigation, Project administration, Resources, Supervision, Writing-review \& editing.

Email: alimujiang31@163.com

Author 4 - Xiao-Li Wang - Formal analysis, Investigation, Resources, Supervision, Validation, Writing-review \& editing.

Email: wxlwgz1112@163.com

Author 5 - Gui-Ping Xu - Conceptualization, Funding acquisition, Investigation, Methodology, Project administration, Resources, Writing-review \& editing.

Email: xgpsyl@126.com 